Diálogo con jóvenes: voces, experiencias y narrativas sobre el suicidio

Luis Carlos A. R. Chow 


\section{Diálogo con jóvenes: voces, experiencias y narrativas sobre el suicidio}

Dialogue with young people: voices, experiences and narratives about suicide
Luis Carlos A. R. Chow

Estudiante antropología social. UNAN-Managua. https://orcid.org/0000-0001-5855-8187 luis.ruchw@gmai.com

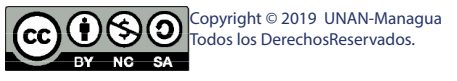

Recibido: 02 Mayo 2019 Aceptado: 10 Junio 2019

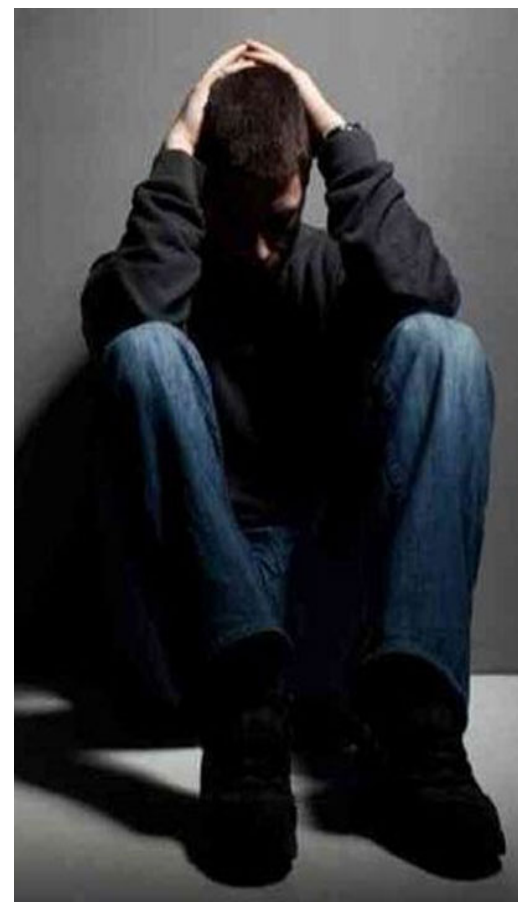

\section{RESUMEN}

En solo el año 2018, se contabilizaron a nivel mundial 800,000 muertes por suicidio. En América Latina el promedio de personas que cometen suicidio es 9.8 por cada 100,000 habitantes. El grupo etario en el que predomina este suceso se encuentra en un rango de 14 - 35 años. En Nicaragua las cifras de suicidios han tomado auge estrepitosamente, siendo el 2017 el año más alarmante con más de 300 casos registrados. Se han identificado las características del comportamiento suicida, habiendo predominancia en las zonas urbanas, entre edades de 12 a 35 años, entre ciudades del área Pacífico y Centro Norte y posteriormente al Caribe Sur. Entre los motivos identificados con los jóvenes que dialogué, destacan conflictos familiares, acoso escolar de tipo agresivo/psicológico, sistemas de creencias que los excluyen, discriminan y condenan; sistemas culturales de carácter patriarcal, procedimientos pedagógicos que pasaron por alto las situaciones específicas y no identificaron un patrón de conducta anormal, enfermedades mentales cuyos padecimientos se desconocían, imaginarios colectivos no compatibles con su identidad, y sobre todo, problemas de autoestima. El objetivo del artículo es acercar al lector sobre el fenómeno del suicidio desde las voces y narrativas de sus protagonistas, al igual que describir el comportamiento epidemiológico en la región latinoamericana y nacional.

\section{ABSTRACT}

In 2018, 800,000 people die and were counted worldwide by OMS. In Latin America, the average number of people who commit suicide is 9.8 per 100,000 inhabitants. The age group in which this event predominates is in a range of 14 to 35 years. In Nicaragua, suicide figures have boomed sharply, with 2017 being the most alarming year with more than 300 registered cases. The characteristics of suicidal behavior have been identified, being predominantly in urban areas, ages between 12 - 35 years

\section{PALABRAS CLAVE}

Suicidio, estigmas sociales, imaginarios colectivos, narrativas y voces.

\section{KEY WORDS}

Suicide, social stigmas, collective imaginary, narratives and voices. 
between cities in the Pacific and North Central area and subsequently to the South Caribbean. The reasons identified with young people whom I dialogue with, arise different variables that combine, between specific family conflicts, aggressive / psychological bullying, belief systems that exclude, discriminate and condemn; patriarchal cultural systems, pedagogical procedures that overlooked specific situations and without identification of a pattern of abnormal behavior, mental illnesses whose illnesses are unknown, collective imaginary not compatible with their identity, and over all self-esteem problems. The objective of the article is to bring the reader closer to the phenomenon of suicide from the voices and narratives of its protagonists, as well as to describe the epidemiological behavior in the Latin American and national region.

\section{Introducción}

suicidio es una realidad de la que no estamos alejados o exentos. Las cifras
arrojadas por distintas instituciones como el Instituto de Medicina Legal o el
Ministerio de Salud en Nicaragua demuestran su notable ascenso en nuestro país.

Víctor Gómez, médico general, logró registrar en su trabajo monográfico Comportamiento epidemiológico del intento suicida en Nicaragua; cerca de 800 intentos suicidas entre 2011 y 2015. Mientras el mapa de la salud diseñado por el Ministerio de Salud, demostró que más de 300 suicidios tuvieron lugar en el año 2017. La cifra aumento $41.3 \%$ desde el último dato arrojado en 2014 por el director del Instituto de Medicina Legal, en donde la cifra rondaba los 140 suicidios.

Nicaragua registra la tasa más baja (3 por cada 100,000 habitantes) en comparación al promedio de la región: 9.3 por cada 100,000 habitantes. Los grupos etarios predominantes rondan entre los 15-35 años.

Desde el presente artículo describo el comportamiento del suicidio en cifras regionales y nacionales, con el fin de que se logre visualizar un escenario en constante cambio; esto como un primer momento. Como segundo momento, pretendo acercar las experiencias desde las narrativas de los jóvenes que han intentado suicidarse a usted como lector, de manera que el análisis del discurso sobre la motivación del suicidio se aborde en un espacio más particular.

Dialogué con 3 jóvenes entre 16 y 18 años a quienes conocí años atrás, bajo una estructura metodológica abierta, como es el diálogo semiestructurado, siendo esta técnica el eje metodológico del artículo; al igual que el respaldo bibliográfico de fuentes especializadas en el tema.

\section{Material y método}

El presente artículo cuenta con dos momentos metodológicos claves. El primer momento metodológico es la revisión bibliográfica de fuentes como investigaciones monográficas realizadas en Nicaragua por parte de médicos de la Facultad de Ciencias Médicas de la Universidad Nacional Autónoma de Nicaragua, UNAN-Managua. También se indagó artículos de revistas médicas, boletines epidemiológicos y notas de prensa sobre la situación del suicidio a nivel latinoamericano, con el fin de abordar un contraste entre la situación del suicidio a nivel nacional e internacional. Este primer momento es el eje macro descriptivo del artículo.

El segundo momento, es el diálogo semiestructurado con 3 jóvenes que han intentado cometer suicidio. Cabedestacarquedialogarcon losjóvenessobreestostemasesun reto, porquelasituación emocional que trastoca sus vidas los cohíbe al detallar, relatar o describir ciertos acontecimientos. 
Tabla 1. Matriz de diálogo semi-estructurado

\section{Matriz de diálogo semi-estructurado}

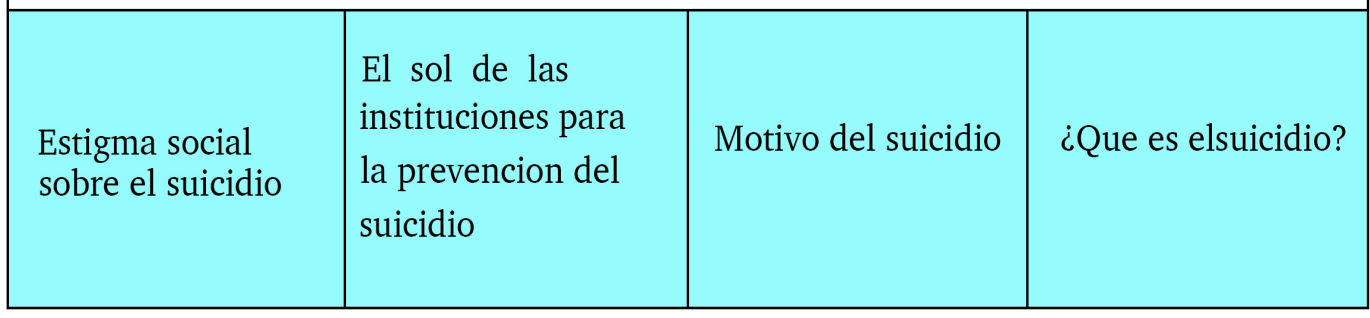

Fuente Propic

El orden de los temas se diseñó de tal manera que el método fuera menos invasivo o rudo, de modo que los primeros temas se establecieron para que los jóvenes se familiarizaran con la actividad y construir empatía.

\section{Ruta metodológica}

El abordaje del tema responde a motivos personales. Como paciente recurrente de la unidad de salud mental en el Hospital Psicosocial Nicaragüense "José Dolores Fletes", me he familiarizado con distintos pacientes es estos últimos 3 años, todos con narrativas cotidianas asombrosas, y ciertamente tristes también. Sin embargo, en la mayoría de momentos en que dialogaba con ellos mientras aguardábamos nuestros turnos de atención, abordábamos el tema, y es cuando tome interés sobre el asunto.

¿Es acaso el suicidio una realidad latente en Nicaragua? ¿Cómo nos encontramos respecto a datos regionales y globales? ¿Qué motiva a un joven a tomar la decisión de suicidarse? ¿Qué significa el suicidio para las personas que lo cometen?

A principios del año 2019, trabajé paralelamente mi investigación monográfica sobre las narrativas de la violencia en los barrios orientales, mientras indagaba datos oficiales sobre el suicidio y el comportamiento epidemiológico nacional respecto al comportamiento regional Latinoamericano, encontrando vacíos respecto a registro, sub-registro, seguimientos y sistematización de datos sobre el fenómeno. Por tanto, como primer objetivo me di a la tarea de ordenar los datos existentes, triangular las cifras oficiales entre el Ministerio de Salud y Medicina Legal respecto a cifras de informes monográficos realizados por médicos de la UNAN-Managua, de manera que pueda construir un panorama general sobre el fenómeno.

Como segundo objetivo para realizar el artículo, comente mi proyecto con los jóvenes con quienes tenía contacto en este espacio de salud, invitándolos a ser parte como informantes clave. Hablé con 17 jóvenes entre 15 y 23 años, sin embargo, enfrentarse con sus realidades fue un verdadero obstáculo para formar parte de la investigación.

El grupo final se redujo a 3 jóvenes entre 16 y 18 años, quienes accedieron en consenso reunirse conmigo el día miércoles 25 de marzo del año 2019. Decidí por motivos de espacio y comodidad realizar el diálogo en las inmediaciones del Parque Japonés en la ciudad de Managua, cuyas características respecto a ambiente y seguridad brinda cierta sensación 
de bienestar y fluidez para expresar ideas que ciertamente son complicadas de narrar.

Los jóvenes rondan entre los 16 y 18 años. Dos hombres y una mujer. Por motivos personales se maneja en seudónimo a sus identidades:

Tabla 2. Características de los informantes.

\begin{tabular}{|c|c|c|c|c|c|}
\hline Seudónimo & Edad & Nivel Academico & Religión & $\begin{array}{c}\text { Intentos } \\
\text { de } \\
\text { Suicidio }\end{array}$ & Enfermedad ${ }^{1}$ \\
\hline P.J. & 16 & Est. Secundaria & Crist-Católico & 2 & Depresión \\
\hline A.A. & 16 & Est. Secundaria & Crist-Católico & 3 & $\begin{array}{c}\text { Trastorno } \\
\text { Limite de } \\
\text { Personalidad }\end{array}$ \\
\hline J.O. & 18 & Est. Universitario & Crist-evangélico & 1 & Depresión \\
\hline
\end{tabular}

Como tercer objetivo, meses de anticipado, me propuse relacionar a los participantes entre sí, permitiendo construir procesos de empatía, de manera que el día establecido para reunirnos fuese menos abrumador. Por tanto cree un grupo en una red social para presentar a cada uno de ellos, a tal punto que la reunión adquirió un significado más cálido y humano.

\section{Discusión de resultados}

\section{El suicidio en Latinoamérica: ¿Una alarma para Nicaragua?}

La Organización Mundial de la Salud en el año 2018, refiere que "cerca de 800000 personas se suicidan cada año - a nivel mundial, y por-cada suicidio, hay muchas más tentativas de suicidio" (OMS, 2018, pág. 2).

En el mismo informe, se señala que el grupo etario de 15 a 29 años es el más afectado, siendo esta acción la segunda causa principal de defunción en este grupo.

En particular, Latinoamérica cuenta con tasas de suicidio de 9.8 por cada 100 mil habitantes, siendo Guyana el país que lidera la lista con 29 por cada 100 mil habitantes; seguida de Bolivia 18.7 y Uruguay 17. Según un informe de la Organización Panamericana de la Salud, afirma que el principal factor de riesgo referente a este fenómeno son "las barreras para obtener atención de salud" (OPS, 2014, pág. 18) Detalla que los grupos etarios afectados en Bolivia abarcan desde los 5 a 14 años, mientras que Uruguay estima el rango de 14 a 24 años. 
La OMS en su informe del 2014 titulado: "Prevención del suicidio: un imperativo global", recomienda:

Aumentar la sensibilización respecto de la importancia del suicidio y los intentos de suicidio para la salud pública, y otorgar a la prevención del suicidio alta prioridad en la agenda mundial de salud pública. También procura alentar y apoyar a los paises para que desarrollen o fortalezcan estrategias integrales de prevención del suicidio en el marco de un enfoque multisectorial de la salud pública" (OMS, 2014, pág. 93).

¿Cuáles son las principales causas del suicidio en dichos grupos etarios? Según informes publicados, artículos e investigaciones realizadas sobre el fenómeno, una de las principales causas es el componente socio-económico como factor determinante y luego el componente emocional como factor condicionante.

El estigma que rodea el fenómeno del suicidio lo hace casi incomprensible, por tanto presentare los componentes biológicos que actúan antes y durante el suicidio, para luego plantear ciertos condicionantes sociales.

Hay aspectos neurobiológicos que caracterizan el suicidio. Un artículo publicado por los doctores Alfonso Martín del Campo, Cristina González y Juan Bustamante, establecen que la base del suicidio se puede detallar en la genética del individuo, al igual que la "disfunción del sistema serotoninérgico (...) como factor predisponente de la conducta suicida" (del Campo, González , \& Bustamante, 2013, pág. 4).

El sistema antes mencionado es el encargado de transportar la serotonina y el genotipo de la enzima hidroxilasa triptófano (TPH), importantes para la estabilidad emocional en el organismo. Según los doctores antes mencionados, el estudio forense dictaminó, que en la mayoría de los jóvenes examinados reportaron la "disminución de la serotonina en la zona del córtex prefrontal y alteraciones en la materia blanca del córtex órbitofrontal izquierdo" (del Campo, González , \& Bustamante, 2013, pág. 4). La zona del córtex prefrontal según los neurólogos Kolb y Wishaw (2006, págs. 1-3) tiene conexiones con el sistema límbico, el hipocampo y el tálamo, importantes para la gestión de conducta, atención, memoria, inteligencia, motivación y estabilidad emocional en el individuo.

Mientras que una afectación en el córtex órbitofrontal representa un cambio radical en las respuestas sociales y la inhibición de conductas poco aceptables, "lo que podría relacionarse con la impulsividad y la conducta en pacientes con trastorno bipolar, depresión o trastorno límite de la personalidad" (del Campo, González , \& Bustamante, 2013, pág. 4). Es decir, que los jóvenes que se suicidaron tenían en mayor medida alteraciones patológicas no tratadas adecuadamente o quizás nunca tratadas, además de entender que el comportamiento suicida previo a consumar el hecho presenta conductas alteradas de la conciencia, por lo que la lógica racional de buscar ayuda es improbable, mas no imposible.

Cabe destacar que sería incorrecto considerar que todos los que se suicidan son enfermos mentales. El suicidio no depende únicamente de factores bioquímicos, si no, de condicionantes y estímulos externos en constante simbiosis y resistencia respecto a las emociones y el cómo reaccionamos ante el medio que nos rodea. Por tanto, es válido reflexionar ¿Cómo es posible tratar o intervenir a alguien que nunca se presenta ante el sistema de salud para ser adecuadamente tratado? Las experiencias de Uruguay, Bolivia y Argentina son interesantes 
de analizar. Los tres países implicados desarrollaron una medida de acercamiento entre la institución de salud y el afectado mediante a líneas telefónicas gratuitas. Los proyectos fracasaron, el rango de usuarios era mínimo y los suicidios escalaban vertiginosamente.

La principal actitud de un suicida es la desesperanza, el desánimo. Es lógico entender que alguien en ese estado alterado de conciencia no recurra por ayuda, en tal caso, quienes deben detectar cualquier cambio de conducta o temperamento emocional son los padres o los familiares que componen el núcleo familiar cercano al individuo, no obstante, según la OPS (2014) más del 70\% de los casos de suicidio tienen como referente la desintegración del núcleo familiar por violencia intrafamiliar, o abuso sexual por parte de un familiar; entonces ¿Cómo intervenir en tales antecedentes?.

Parece que el suicidio no es un tema que derive una acción deliberada del momento, sino un proceso de acontecimientos que conllevaron a tomar una decisión final por parte del individuo, y a lo largo de dicho proceso ni siquiera estuvo presente el buscar ayuda en la institución de la salud más próxima, si el paciente no acude a las instituciones, el Estado no es garante para resolver, intervenir o apoyar su situación, por tanto el sujeto se encuentra desprovisto tanto de apoyo moral y familiar como del apoyo institucional.

Otro de los posibles filtros para una probable intervención de los actos de suicidio, es la institución educativa; sin embargo, regresamos al punto de partida inicial ya que según el informe de la OPS (2014) el $25 \%$ de los jóvenes que cometieron suicidio han sufrido acoso escolar.

Es realmente impactante e irónico reflexionar que las estructuras sociales como la familia y la escuela, que se suponen están diseñadas para la formación, protección y educación del niño en el campo cultural e institucional sean, de cierto modo los detonantes de este comportamiento, dando como resultado entender el suicidio como un fenómeno complejo y transdisciplinario.

\section{Datos del suicidio en Nicaragua}

En Nicaragua, las cifras del suicidio en comparación con la región Latinoamérica son relativamente bajas. Según datos de la OMS en conjunto a la $0 \Gamma s$, en su informe "El suicidio según vigilancia epidemiológica" afirma que:

"Enelperiodo1997al2012fallecieron5,673personasporLesionesautoinfringidasintencionalmente, siendo el 2010 el año con mayor número de decesos. Proporcionalmente, de cada 100 defunciones totalesregistradasen elpais, 2 son porlesiones autoinfringidasintencionalmente. Cabeseñalarque de cada 10 defunciones por suicidios, 8 son del ámbito urbano y 2 del rural"(OMS, OPS, 2016, pág. 2).

Éste informe señala que los departamentos con mayores índices son: Managua 26\%, Matagalpa 13\%, Chinandega 11\%, León $8 \%$ y Jinotega 6\%. Regiones del occidente-norte del país y el área capitalina.

El director del Instituto de Medicina Legal de Nicaragua a través de una nota de prensa emitida por el Ministerio de Gobernación afirma que "en el 2012 se registraron a nivel nacional 148 muertes por suicidio, en el 2013 aumentó a 173" (Duarte Castellón, 2014, pág. 7).

Un estudio monográfico realizado por el doctor Víctor Gómez, titulado Comportamiento epidemiológico del intento suicida en Nicaragua, informó que entre el 2011 y 2015, identificó 
886 casos de intento de suicidio, siendo el grupo etario predominante entre 18 - 35 años. Los casos de intento de suicidio identificados ocurrieron en la Región Autónoma del Caribe Sur y Carazo, y las tasas de morbilidad más altas de suicidios consumados se registraron en los departamentos de Carazo y Madriz (Gómez, 2017, pág. 12).

En 2017 según el mapa de la salud mental diseñado por el Ministerio de Salud, detalló que habían cometido suicidio 355 personas, y en 2018 se contabilizaron $224^{2}$ casos. Siendo identificado el grupo etario predominante entre 15 - 30 años ${ }^{3}$.

En términos macro, he logrado dibujar un escenario latinoamericano para detallar la realidad nicaragüense, demostrando como los casos han ido tomando auge, los departamentos con mayor incidencia, el rango de edad predominante y el comportamiento cuantitativo según el año; no obstante, los motivos por los cuales una persona decide cometer suicidio son ambiguos y no están debidamente registrados.

Por tanto, el siguiente capítulo tratara más a fondo sobre el fenómeno del suicidio desde sus protagonistas.

\section{Diálogo con jóvenes: conductas suicidas y conductas autolesivas}

Antes de iniciar el conversatorio, les pedí a los informantes que pensaran en palabras, ideas, acciones o cualquier situación imaginaria relacionada al suicidio, incluso su significado desde sus pronix perspectivas. El resultado fue el siguiente:

\section{"Escape" "Paz" "impulsos y sensaciones" "Siento como si no fuese yo mismo" "Me siento un títere"}

Figura 1 Imaginario Colectivo sobre lo que significa suicidio. Managua, 2019.

\section{C'and's hablamos de suicidio}

Nunca hablamos de los motivos que nos impulsaron a tomar la decisión, es un tabú (...) creen que uno quiere llamar la atención, y no es así, yo en mi caso quiero detener esto, este dolor. No consigo detenerlo, es como si te comiera lentamente y la unica forma de estar en paz, de estar tranquilo, es muerta... Siento pena, me avergüenzo con mi familia por lo que hice, sé que ellos quieren comprenderme, pero ni yo logro bacerlo"

\footnotetext{
2 Cifras del Mapa de la Salud, de la página web del Ministerio de Salud, MINSA: http://mapasalud.minsa.gob.ni/mapa-de-padecimientos-de-salud-de-nicaragua/

3 Cifra del Boletin Anuario 2018 del sitio web del Instituto de Medicina Legal, IML https://www.poderjudicial.gob.ni/pjupload/iml/pdf/Anuario_2018.pdf 
Es fácil juzgar a alguien que toma esa decisión; pero ninguno tiene los huevos de preguntar ¿Cómo te ayudo? ¿Estás bien? (...) te consideran loco, que perdiste la fe, que te vas al infierno (...) No comprenden lo fuerte que son sus palabras (...) yo pienso en lo que me dicen toda la noche, es agobiante ¿Cómo poder vivir asi?”

Podemos destacar el poder del imaginario colectivo para desmotivar, enajenar y someter un constante estado culpabilidad ante las personas que intentan cometer suicidio. Desde las posturas religiosas se discrimina al joven; la religión como un instrumento de victimización.

Como podemos analizar son jóvenes creyentes, cuya religión no admite este acto, y es considerado como una aberración y un atentado contra la vida y la creación de Dios. A este punto su religión se convierte en un espacio de culpa, pues en lugar de encontrar consuelo o alivio ante el constante sufrimiento que aseguran experimentar, se encuentran en cambio con posturas radicales de condenación.

"Te enfrentas a esos comentarios, esas miradas, y luego llegás a tu casa donde no te comprenden en lo absoluto (...) mi madre me reclama muchas veces que no pongo de mi parte, que solo estoy ahi echado [acostado]sinhacernada:peroesquenotengomotivaciónalgunadehaceralgo;salirdelacamaesyapor siunlogroparami, siento quefuerademicuartosoy un extraño, yen lascallestodostemiran, tejuzgan"

El estado permanente de tristeza, soledad y culpa se reflejan en su cotidianidad: falta de motivación por cumplir roles en el hogar, relaciones sociales en conflicto o ausente, constante inasistencia en sus centros escolares.

"Es como si hubiera un botón en mi cabeza, un botón de automático, no siento motivos para salir y hablar con mis amigos, no siento motivo alguno para ir a clases. Hay momentos en que me siento bien solo, el silencio y nada más; pero también hay momentos en que soy diferente y me sorprende"

Estos momentos de eutimia son sumamente necesarios para las personas con síntomas depresivos o bipolares; son momentos que permiten sentar un dialogo; sin embargo, el mal manejo de las situaciones, puede agravar las etapas depresivas. El poder de las palabras y su acción para motivar es una capacidad necesaria tanto en familiares, amigos o personal médico que trata constantemente con estos jóvenes.

¡ya te va pasar! ipensá positivo! ¡ a mi me pasó eso y blah blah! Esas cosas me hacen sentir frustrado, enojado conmigo mismo. No es algo que no quiera superar, decime ¿quién quiere estar triste todo el tiempo? Es absurdo (...) ipone de tu parte! ipedile a Dios! Solo me dan ganas de alejarme, me hacen sentir inútil, como si no quisiera hacerlo, pero es que lo intento y nada funciona..."

Se preguntarán quizás los motivos por los que estos jóvenes tienden a expresarse de manera fatalista y también las razones por las que intentaron cometer suicidio. Las experiencias son particulares, pero logré encontrar un punto en común.

A medida que los jóvenes expresaban sus motivaciones, siempre coincidían en un acontecimiento familiar que los impulsó a tomar su decisión; sin embargo, detallaríamos ese momento como el detonante. Previo a todo el proceso, el eje principal se encontraba en su autoestima, el cómo se auto-describían. 
Adjetivos como: inútil, maricón [homosexual], flojo [holgazán], bruto [tonto]; son los insumos que componen el imaginario que construye su identidad.

J. O me permitió comentar su caso: es un joven homosexual, que desde los 12 años descubrió sus gustos que contradicen las normativas de la religión que profesa y las normas culturales de su comunidad y familia. El constante estrés que experimentaba al sobre pensar distintos escenarios en donde sus padres lo discriminaran, golpearan o rechazaran; sumado al constante rechazo y agresión de sus compañeros en el colegio, motivaron a tomar tal decisión

Mis padres se sorprendieron cuando me encontraron teniendo relaciones sexuales [con otro joven]... no podian comprender que pasaba, y cuando me estabilicé intentaron o más bien, me forzaron a que dijera por qué lo hice. Solo me dieron atención hasta que estaba ahí, ninguno notó que odiaba ir al colegio, que estaba teniendo malas notas"

Ante un suicidio, existen alarmas previas. Este es el papel que tienen las instituciones escolares, pero sobre todo los padres. El silencio de la institución ante la problemática que J.O experimentaba y la lectura poco preocupada sobre su rendimiento académico provocaron el primer escenario de riesgo. La poca confianza ante sus padres para abordar el conflicto es el segundo escenario de riesgo, lo alarmante es que ninguno notara los cambios o comportamientos de su hijo. No logré descubrir que sucedía en el núcleo familiar para que sus padres no notaran esta situación.

Les pregunté a los tres informantes, si previo al hecho, habían considerado en algún punto acudir a un psicólogo o un doctor. Los tres lo negaron. Cuestioné el porqué:

Loqueyoentendiaantesesquesiibasconelpsicólogoerasloco(...) ademássupedeunacompañeraquesu mamálaatendian;peroqueibacada 3 meses (...) nomemirabasoportandoestasituaciónparahablar con alguien que no conozco en 3 meses, no confió en mis padres ¿Quéme haría confiar en el doctor??"

La empatía es el pilar fundamental para construir una relación. Si un hijo no tiene la capacidad para expresar ante sus padres sus sentimientos, sus gustos, lo que piensa o lo que intenta hacer es debido a toda una amalgama de ideologías y creencias que los jóvenes han construido frente a las distintas posturas que los identifican como tal. Este entramado de situaciones socio-culturales implica el sobrellevar estos escenarios de rechazo constante y emprender procesos de resistencia y catarsis ante el asedio físico y emocional constante.

A veces siento vergüenza de mi mismo, me siento impotente de no poder cambiar nada en mi (...) cuando no puedo más, y me siento como que exploto decido cortarme en el brazo (...) algunas veces también uso el encendedor para quemarme (...) son momentos en que aún no decido suicidarme realmente, solo deseo sentir algo, aunque sea dolor ${ }^{10 "}$

Ante esa aclaración A.A y J. O afirmaron que también tendían a auto-infligirse daño, J.O en menor medida queA.A, siendo una escalavalorativa de $1-10$ (1 como no tan frecuentey 10 como frecuente):

9. A.A. Diálogo semiestructurado. $25 / 03 / 2019$.

10. P.J Diálogo semiestructurado. 25/03/2019. 


$$
\begin{array}{ccc}
\text { A.A:8 } & \text { J.O:5 } & \text { P.J:7 } \\
\begin{array}{c}
\text { Laceraciones en brazos y } \\
\text { piernas }
\end{array} & \text { Laceraciones en brazos } & \text { Laceraciones y uso de fuego }
\end{array}
$$

Tabla 3 Escala valorativa respecto a frecuencia de auto-lesión y métodos empleados. Elaboración propia, Managua 2019

Las conductas auto-lesivas son mecanismos de defensa y resistencia frente a estímulos externos; están pensados para sobrellevar ciertos acontecimientos cotidianos e incluso fuera de lo cotidiano. Las auto-laceraciones cumplen dos objetivos en concreto; primero para la persona que se autoinflige es un método de catarsis, que a su vez permite visibilizar a los demás, generalmente padres o amigos, el acto cometido, provocando cierta preocupación. alguien capte el mensaje y posiblemente sea en quien pueda confiar. También existen situaciones en los que esta conducta es totalmente indiscreta y los sujetos no buscan más atención, simplemente la decisión final.

Las victimas del suicidio inconscientemente interiorizan los discursos que contradicen su identidad (en el caso de J.O.) y su única salida es el suicidio. El miedo ante el rechazo de los padres o incluso la posible agresión de los padres es mucho mayor a perder la vida.

Y es que el suicidio más allá del acto de auto infringirse daño con el fin de morir, es desde la perspectiva de sus autores, la salida, el escape a sus problemas. La desesperanza y el estado depresivo constante, no les permite visualizar un futuro mejor, o siquiera un futuro y la lucha constante con su entorno; pero sobre todo, consigo mismo es una tortura.

El conflicto entre vida y muerte es un aspecto necesario de retomar. Mientras el discurso de la vida lo posicionan al margen de lo fatálico, la muerte simboliza el escape. Desde el análisis funcional, el vivir es un proceso en el que se cumple un rol específico y un papel colectivo; ahora, retomando los adjetivos que construyen la imagen de los jóvenes, "inútil" es el que adjetivo que más refieren en todo momento. Si analizamos todas las citas antes expuestas encontraremos la dificultad de proyectar a futuro.

De cierta manera el miedo a vivir es el miedo al fracaso, y el suicidio es el escape para evitar afrontar a toda costa ese miedo. La evasión de afrontar el conflicto. No obstante, son sentimientos que cualquier persona en algún momento de la vida puede llegar a cuestionar; cabe destacar que no todos responden de maneras similares y hay que recordar que estos jóvenes han sido diagnosticados ya sea con depresión o trastorno límite de personalidad; lo que implica el hecho de tender hacia una postura o respuesta totalmente distinta o incluso impulsiva para responder a esta situación cotidiana.

El miedo al rechazo, abandono, y frustración son sentimientos reales que afectan la cotidianidad y las relaciones sociales y entenderlas es un pilar fundamental del cual las instituciones a cargo deben responsabilizarse, al igual que los núcleos familiares y comunitarios.

11. P.J Diálogo semiestructurado. 25/03/2019. 
El suicidio... es paz (...) es ese momento donde ya nada importa, donde todo desaparece, sos invisible y el mundo desaparece ${ }^{11} "$

Algunas consideraciones a tomar en cuenta sobre el contexto de cada uno de los chicos para entender la influencia de los espacios y las dinámicas comunitarias y su correlación con el comportamiento suicida es la siguiente:

P.J. habita en el municipio de Ciudad Sandino, una zona altamente vulnerable en materia de seguridad, riesgos sociales como adicción a drogas legales e ilegales, pandillas juveniles y delincuencia. Últimamente han tomado auge algunas denominaciones religiosas cristianoevangélicas.

A.A. es de Villa Fontana, tiene tratamiento privado, la zona en la que reside se considera a nivel social exclusiva; sin embargo, es el que más intentos de suicido ha realizado. En cambio, J.O reside en Villa Venezuela, un barrio con características similares a Ciudad Sandino en el ámbito de vulnerabilidad.

A este punto, se puede afirmar que no hay un motivo predominante entre el contexto y la conducta suicida, sino un motivo condicionante entre las relaciones intrapersonales con el entorno familiar de cada individuo, y como un segundo plano el contexto incide como una carga de estress. Por lo que es necesario recomendar a futuros estudios monográficos el comprender las dinámicas familiarese institucionales decadauno de los sujetos que intentan suicidarseysu posturafrenteasu comunidad. Otra consideración a tomar en cuenta son los métodos utilizados para cometer el suicidio ${ }^{12}$ :

\section{Seudónimo 1er intento 2do intento 3er intento}

$\begin{array}{ccc}\text { P.J } & \begin{array}{c}\text { Sobredosis de } \\ \text { fármacos }\end{array} & \text { Ahorcamiento }\end{array}$
A.A
Sobredosis de
Auto-laceración y
fármacos
desangrado
Sobredosis de
fármacos

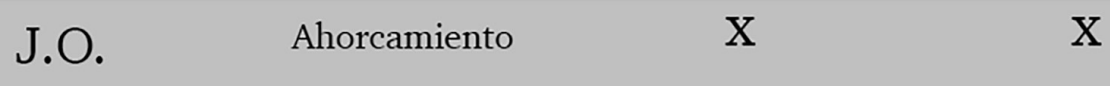

Tabla 3 Mètodos para consumar el suicidio. Elaboración propia, Managua 2019.

Predomina el uso de fármacos para provocar intoxicación, seguido del ahorcamiento y autolesión por desangrado.

\section{Conclusiones}

En el año 2018 se contabilizaron a nivel mundial 800,000 muertes. En américa latina el promedio de personas que cometen suicidio es 9.8 por cada 100,000 habitantes. Los grupos etarios que predominan están entre los 14-35 años. En Nicaragua las cifras son bajas respecto a los datos regionales y globales. Se han identificado las características del comportamiento suicida, siendo predominante en las zonas urbanas, en grupos etarios entre los $15-35$. 
Los motivos identificados con los jóvenes con quienes dialogué surgen por conflictos familiares, acoso escolar de tipo agresivo/psicológico, sistemas de creencias que los excluyen, discriminan y condenan; sistemas culturales de carácter patriarcal, sistemas pedagógicos que no actuaron ante situaciones específicas y no identificaron un patrón de conducta anormal, enfermedades mentales de las cuales no eran conscientes previos a cometer el suicidio, imaginarios colectivos no compatibles con su identidad, y sobre todo problemas de autoestima.

Se identificó el comportamiento auto-lesivo como un mecanismo de resistencia y alivio ante las situaciones y estímulos cotidianos que los estresan; al igual se detalló los métodos recurrentes que los jóvenes utilizaron para cometer el suicidio, predominando el uso de fármacos.

\section{Referencias bibliográficas}

- Del campo, A., González , C., \& Bustamante, J. (2013). El suicidio en adolecentes. Revista Médica del Hospital General de México, 169-233.

- Duarte Castellón, Z. (2014). Instituto de Medicina Legal. Retrieved from Muertes por suicidio en Nicaragua la más baja de la región: https://www.poderjudicial.gob.ni/pjupload/iml/pdf/ suicidios.pdf

- Gómez, V. (2017). Comportamiento epidemiológico del intento suicida en Nicaragua. Retrieved from Repositorio UNAN-Managua : http://repositorio.unan.edu.ni/9730/

- Kolb, B., \& Wishaw, I. (2006). Neuropsicología humana. Madrid: Editorial Médica Panamericana.

- OMS.(2014).Prevencióndelsuicidio:unimperativoglobal.RetrievedfromOrganizaciónMundial de la Salud : https://apps.who.int/iris/bitstream/handle/10665/136083/9789275318508_ spa.pdf;jsessionid=B3A4F5FFC198915C9DF4F1F35A8F502C?sequence $=1$

- OMS. (2018, Agosto 24). Organización Mundial de la Salud. Retrieved from https://www. who.int/es/news-room/fact-sheets/detail/suicide

- OMS, OPS . (2016). WHO. Retrieved from El Suicidio según Vigilancia Epidemiológica: https://www.paho.org/nic/index.php?option=com_docman\&view=download\&category_ slug=datos-y-estadisticas\&alias=713-boletin-informativo-el-suicidio-segun-vigilanciaepidemiologica\&ltemid $=235$

- OPS. (2014). Organización Panamericana de la Salud. Retrieved from Factores y riesgo de suicidio en America Latina : http://iris.paho.org/xmlui/bitstream/hand le/123456789/31167/9789275319192-spa.pdf?sequence=1\&isAllowed=y 


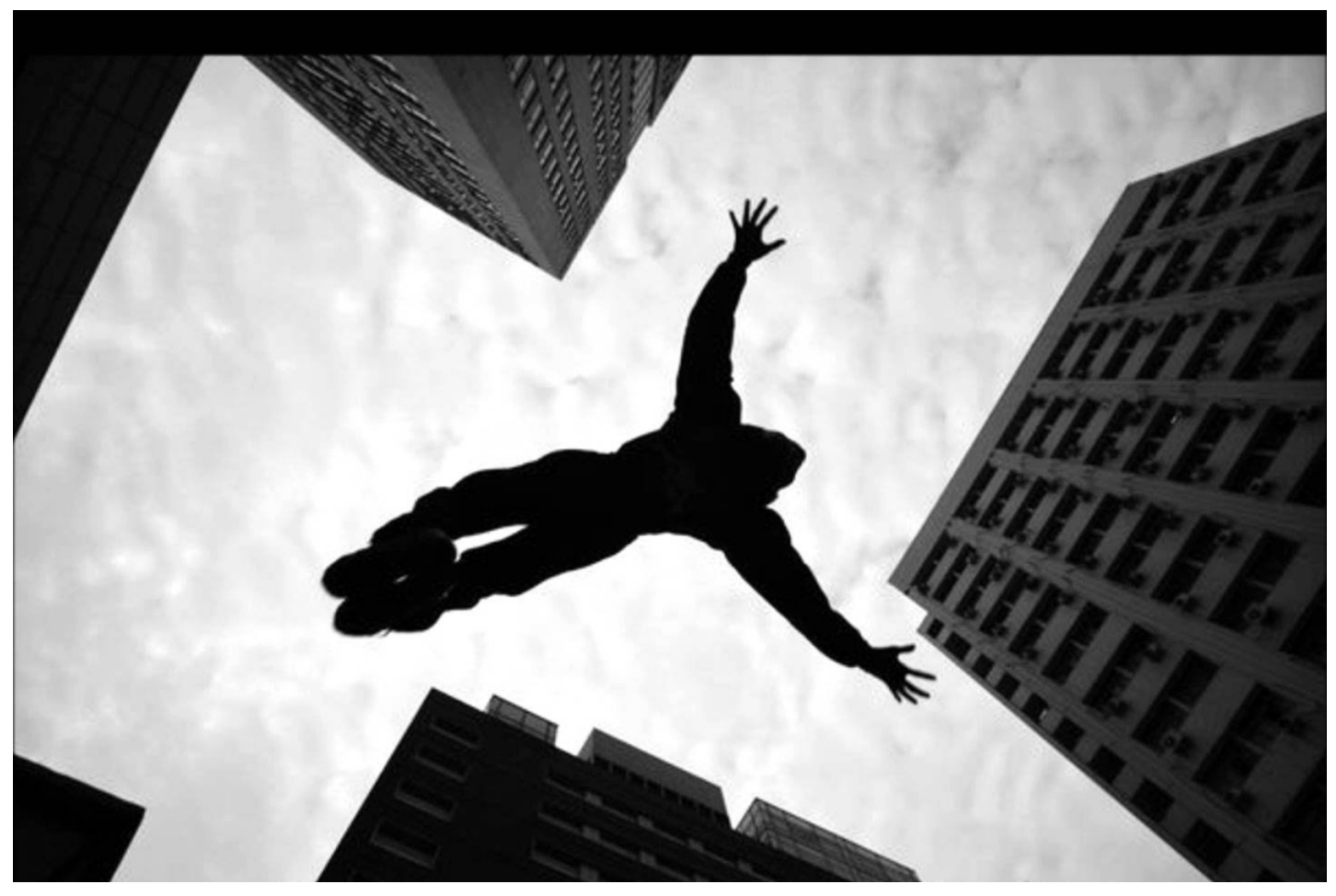

Luis Carlos A. R. Chow

Estudiante antropología social.

UNAN-Managua.

https://orcid.org/0000-0001-5855-8187

luis.ruchw@gmai.com 